\title{
Norois
}

Environnement, aménagement, société

$224 \mid 2012$

Agriculture paysanne, circuits courts, territoires périurbains

\section{L'adaptation des agriculteurs au contexte périurbain}

Une lecture des logiques agricoles à partir du cas de Billom-Saint-Dier (Auvergne)

How do farmers adapt to periurban context? Agricultural rationales at Billom-

Saint-Dier (Auvergne - France)

\section{Marie Houdart, Salma Loudiyi et Alain Gueringer}

\section{(2) OpenEdition}

Journals

\section{Édition électronique}

URL : https://journals.openedition.org/norois/4265

DOI : $10.4000 /$ norois. 4265

ISBN : 978-2-7535-2157-5

ISSN : 1760-8546

Éditeur

Presses universitaires de Rennes

Édition imprimée

Date de publication : 30 septembre 2012

Pagination : $35-48$

ISBN : 978-2-7535-2155-1

ISSN : 0029-182X

\section{Référence électronique}

Marie Houdart, Salma Loudiyi et Alain Gueringer, «L'adaptation des agriculteurs au contexte 


\title{
L'adaptation des agriculteurs au contexte périurbain Une lecture des logiques agricoles à partir du cas de Billom-Saint-Dier (Auvergne)
}

\author{
How do Farmers Adapt to Periurban Context? \\ Agricultural Rationales at Billom-Saint-Dier (Auvergne-France)
}

Marie Houdart $^{\text {a*}}$, Salma LoudiYI ${ }^{\mathrm{a}}$, Alain Gueringer ${ }^{\mathrm{b}}$

\footnotetext{
* Auteur correspondant

a IRSTEA - UMR 1273 Métafort, BP 500085 - 63172 Aubiène cedex (marie.houdart@irsteacemagref.fr) (alain.

gueringer@irsteacemagref.fr)

b PRES Clermont - VetAgro Sup - UMR 1273 Métafort, 89, Avenue de l’Europe, BP 35 - 63370 Lempdes (salma.

loudiyi@vetagro-sup.fr)
}

Résumé : L'article traite des logiques d'adaptation des agriculteurs au contexte périurbain. L'analyse est menée sur un territoire situé à l'est de l'agglomération clermontoise (9 communes de la communauté de communes de Billom-Saint-Dier). L'adaptation des agriculteurs est saisie à partir des composantes productive et commerciale, de la diversification des activités et des logiques de gestion du foncier. Les résultats montrent que les signaux d'adaptation sont faibles en ce qui concerne les composantes productives et les modalités de gestion du foncier. Les logiques d'adaptation s'expriment un peu plus à travers la composante commerciale. La faiblesse des signaux d'adaptation des agriculteurs au contexte périurbain interroge leur capacité d'intégration dans les dynamiques collectives et institutionnelles mettant en lien ville et agriculture dans la zone étudiée.

Abstract: The aim of the present study is to assess the individual and collective adaptation of farmers in rural-urban fringes. The case study has been led on a peripheral area of the city of Clermont-Ferrand (centre of France in the Massif Central). The analysis focuses on production, marketing, diversification of the activities and logics of land's management. The results revealed some weak signals of adaptation. This questions the capacity of farmers to integrate collective and institutional dynamics led by periurban transformations.

Mots clés : Périurbain - agriculture - adaptation - Billom-Saint-Dier

Keywords: Rural-urban fringes - agriculture - adaptation - Billom-Saint-Dier

\section{INTRODUCTION}

L'accroissement démographique des territoires périurbains témoigne de leur attractivité depuis le milieu des années 1960 en France. Plus récem- ment, la croissance endogène des périphéries urbaines succède aux processus de desserrement résidentiel (Cailly, 2010). Plusieurs travaux ont identifié les implications des processus de périurbanisation sur la redéfinition des fonctions 
associées à ces espaces ruraux en périphérie des villes (Bryant et Johnston, 1992). Ils ne sont plus simplement des espaces productifs et agricoles, ils sont le support à la fois de fonctions résidentielles, récréatives et environnementales. Malgré la vigueur de l'étalement résidentiel dans certaines régions, les espaces agricoles et naturels restent dominants dans le paysage et constituent un enjeu de taille dans le processus de périurbanisation. La volonté de limiter la « consommation » de l'espace agricole rencontre des objectifs de valorisation des paysages. À la confluence d'un discours généralisé sur la gestion économe des espaces et de la pluralité des fonctions attribuées à l'agriculture, le rôle assigné à cette dernière pose un ensemble de questions autour des modalités de gouvernance des liens entre ville et agriculture.

Les démarches de planification stratégique intègrent de plus en plus la question agricole dans les modalités de construction des villes durables. La généralisation des Schémas de Cohérence Territoriale (SCoT), l'institution progressive des Périmètres de protection et de mise en valeur des espaces agricoles et naturels périurbains (PAEN) et des Zones Agricoles Protégées (ZAP) constituent autant d'instruments d'action publique propres à intégrer l'agriculture dans le projet urbain et posent la question de sa durabilité (Duvernoy et al., 2005). Cependant, les modalités de cette intégration varient selon les situations locales (Vidal et Fleury, 2009; Loudiyi et al, 2011; Pasini et al, 2012), et plusieurs travaux en soulignent les limites : plus ou moins bonne représentation des acteurs agricoles et de leurs intérêts (Duvernoy, 2002; Vianey, 2005); approche souvent limitée à celle de l'espace agricole (Jarrige et al., 2009; Loudiyi et al., 2010); difficile prise en compte des projets agricoles. Les négociations peuvent s'avérer difficiles et tortueuses (Poulot, 2008; Poulot, 2011 ) et les stratégies individuelles des agriculteurs s'accorder plus ou moins avec les objectifs affichés par le niveau institutionnel.

La question foncière a souvent été une clé d'entrée pour analyser le rapport urbain/rural et expliquer l'érosion des structures agricoles dans le périurbain (Vianey et al., 2006). Le facteur foncier doit toutefois être replacé dans le contexte de dynamiques sociales et de politiques locales qui fondent les stratégies et les jeux d'acteurs. La compréhension des représentations et des logiques d'action des agriculteurs s'avère importante pour saisir les différentes modalités de construction des liens entre ville et agriculture (Bryant, 1997; Le Caro et al., $2010^{1}$ ). S'appuyant sur les résultats d'une enquête conduite sur un espace situé dans la périphérie de l'agglomération de Clermont-Ferrand, cet article propose une lecture des signaux d'adaptation des agriculteurs à un contexte périurbain considéré comme une contrainte mais également comme une opportunité à saisir.

\section{Adaptation, innovation ET VALORISATION DE L'AGRICUL- TURE EN CONTEXTE PÉRIURBAIN : CONTEXTE SCIENTIFIQUE ET GÉOGRAPHIQUE}

\section{Agriculture et périurbanisation}

La notion de valorisation des ressources territoriales postule que l'idéal du développement territorial se concrétise lorsque des acteurs, dans un territoire donné, identifient, spécifient et valorisent des ressources locales en s'organisant et en mettant en œuvre des coordinations autour de ces ressources (Gumuchian et Pecqueur, 2007). Tout territoire dispose d'une variété de ressources locales qui peuvent se transformer en ressources territoriales dès lors qu'un collectif d'acteurs s'organise et engage ce travail de spécification (Bernard et al., 2006; Poulot, 2008).

Dans les territoires périurbains, les pressions exercées sur le travail des agriculteurs sont nombreuses et peuvent complexifier cette possible spécification de la ressource agricole. Ces pressions, ou forces motrices, concernent des échelles et des domaines différents. À l'instar de l'ensemble des agriculteurs français, ceux des aires périurbaines sont soumis aux effets du changement climatique, à la fluctuation des cours des différentes productions, à l'évolution de la PAC, aux nouvelles réglementations agro-environnementales, etc. (Agrimonde, 2009). Au niveau local, les agriculteurs doivent également intégrer les effets de la désectorialisation de l'agriculture. Les modalités d'intégration de l'agriculture dans les projets de territoire et les orientations stratégiques des collecti-

1. Le Caro Y., Pierre G., Margetic C., 2010. Le statut social de la terre agricole dans un espace rural en voie d'urbanisation, le Coglais (Bretagne). Intérêt et limites d'enquêtes par groupes de discussion, communication au colloque ASRDLF, Aoste, septembre 2010, 20 p. 
vités territoriales en lien avec les nouvelles demandes sociales (demandes relatives au paysage et à l'environnement, émergence des questions de gouvernance alimentaire) en sont des exemples. À ces pressions générales, s'ajoutent les spécificités des zones périurbaines : proximité au marché des emplois urbains, à la technologie, compétition pour l'utilisation du sol, rente de localisation (Bryant, 1997).

Malgré toutes ces contraintes, le périurbain peut être vu comme espace de redéfinition de l'exercice de l'activité agricole (Dufour et al., 2003; Germain et al., 2006; Rouget, 2008). En effet, les dynamiques locales des zones périurbaines, en interaction avec une multiplicité d'espaces sociaux, prennent une nouvelle place dans les processus d'innovation (Chiffoleau et al., 1999²). La capacité à innover permet aux agriculteurs de faire face aux différentes pressions qu'ils subissent et à l'évolution des contextes socio-économiques. Les ajustements, induits par un nouvel environnement ou un contexte changeant, peuvent aussi s'exprimer par des adaptations relevant davantage de réponses réactives que de réponses anticipées. Ainsi, les signaux d'adaptation des agricultures périurbaines peuvent être vus comme un potentiel existant pour une intégration durable à la ville; la faiblesse de ces signaux constituent à l'inverse un facteur de risque à la fois pour les agricultures et pour les villes.

À propos des territoires périurbains, Debarbieux (2005) évoque une « ruralité en invention » qui a amené nombre de chercheurs à se pencher sur les spécificités de l'agriculture périurbaine (Bryant, 1997). Ces travaux dégagent ainsi des formes d'agriculture spécifiques de ces espaces périurbains (Jarrige, 2004 ; Soulard et Thareau, 2009), qui s'accompagnent bien souvent d'adaptations sociales et de nouvelles formes de coordination entre les différents acteurs du monde agricole (Bernard et al., 2006). Dans tous les cas, l'adaptation et l'innovation se font également dans les pratiques du quotidien, parfois de façon formelle dans le cadre des groupes professionnels locaux et, plus largement, dans des réseaux dont la création est impulsée par le phénomène de périurbanisation (Darré, 1986).

2. Chiffoleau Y., Dreyfus F., Touzard J.-M., 1999. Systèmes d'action innovante, systèmes localisés de production : une approche pluridisciplinaire de la transformation de la viticulture en Languedoc-Roussillon, communication à l'École-chercheur «Économie régionale, économie spatiale », INRA, Le Croisic, 8-10 décembre 1999, ACTIV, INRA-SAD Montpellier 1
En définitive, dans le contexte particulier d'un processus de périurbanisation, les agriculteurs s'adaptent aux mutations en cours en termes de réactivité aux contraintes, mais également en termes de valorisation et d'exploitation de nouvelles potentialités offertes. Ces formes d'adaptation peuvent s'opérer dans différents domaines et de différentes façons : adaptation passive ou réactive, innovations dans les pratiques, etc. (Bertrand et Roussier 2010; Leger et al., 20113).

Dans cet article, nous nous intéressons aux signes d'adaptation des agriculteurs au contexte périurbain et de ses effets en termes de valorisation des ressources agricoles. Nous nous appuyons sur l'hypothèse que l'adaptation recouvre différents domaines et qu'elle est variable suivant ces mêmes domaines. Nous l'abordons à l'échelle des exploitations agricoles et à partir des composantes productives, commerciales et de gestion du foncier (Jarrige, 2004). Cette clé de lecture s'appuie sur les dynamiques propres à une agriculture en lien avec la ville. D'une part, la proximité d'un nombre de consommateurs peut représenter un potentiel de commercialisation des productions agricoles, que les agriculteurs peuvent valoriser. D'autre part, la question du foncier agricole peut être considérée comme une contrainte lorsque l'incertitude, la pénurie ou le prix des terres jouent un rôle défavorable dans le développement ou le maintien de l'agriculture.

\section{Billom-Saint-Dier : visage rural, fonctionnement périurbain}

La communauté de communes du Pays de BillomSaint-Dier se situe à l'est de l'agglomération clermontoise. Elle est située à l'interface de deux entités morphologiques : la Limagne des Buttes et le massif du Livradois. À l'inverse de la Grande Limagne, formée par de larges couloirs de basses terres, la Limagne des buttes est «montueuse » (Fel, 1983). Elle correspond à des plaines vallonnées, ponctuées par des necks. La grande culture y domine et marque nettement le paysage. Lorsqu'elles sont bien exposées, les buttes sont occupées par la vigne. Les sommets sont parfois boisés et occupés par des

\footnotetext{
3. Leger C., Alavoine-Mornas F., Lanquetin J., 2011 . Effets des grands projets d'aménagement sur les dynamiques des exploitations agricoles périurbaines, communication au colloque «Dynamiques des espaces ruraux et périurbains : Le développement durable face à la mondialisation », université d'Évry Val d’Essonne, 27-28 octobre 2011.
} 
édifices remarquables (châteaux, tours et chapelles). Ces caractéristiques paysagères valent parfois à la région le surnom de «Toscane auvergnate ».

Tout en se fondant historiquement sur la polyculture, la vie rurale de ce territoire a été marquée par les vicissitudes de la vigne (Derruau, 1949). Le phylloxera, survenu tardivement dans les campagnes auvergnates, a ruiné le destin de cette vigne, mais on perçoit encore sa présence sur les coteaux les plus ensoleillés. La diversification des productions est intervenue tôt et la culture de l'ail a été favorisée pour son « rapport immédiat ». Elle renaît aujourd'hui à travers des démarches de production de qualité (Ail d'Auvergne, Ail de Billom).

En direction du sud-est, les premiers contreforts du Livradois réunissent des plateaux surélevés où s'interpénètrent vallons agricoles et versants forestiers. Cette entité présente un caractère bocager plus prégnant, aujourd'hui propice à l'élevage, là où, historiquement, la petite culture subsistait. L'habitat y est organisé en hameaux groupés, en nombre élevé par commune.
L'influence de l'agglomération clermontoise, d'abord réduite aux communes les plus proches, se diffuse sur l'ensemble du territoire depuis les années 1990. D'après le dernier recensement de 2007, la communauté de communes de Billom-Saint-Dier compte près de 12000 habitants, soit une progression annuelle de 1,6\% depuis 1999 (tableau 1). Le rythme annuel de la croissance démographique est ainsi cinq fois plus rapide que celui du département $(0,5 \%)$. Certaines communes tirent profit du desserrement urbain et connaissent des hausses relativement importantes de leur population : Chas ou Reignat, localisées dans la Limagne des Buttes, enregistrent ainsi des rythmes de croissance supérieurs à $3 \%$ par an depuis 1999. Leur densité démographique en est affectée et l'emprise spatiale de cette urbanisation devient sensible.

Les moteurs de cette vigueur démographique sont d'abord des soldes migratoires bénéficiaires, relayés par les soldes naturels, quoique largement inférieurs. La pression urbaine se traduit par l'importance de l'habitat individuel $(87,3 \%$ en 2007).

\begin{tabular}{|c|c|c|c|c|c|c|}
\hline & $\begin{array}{l}\text { Population } \\
\text { en } 2007\end{array}$ & $\begin{array}{c}\text { Densité en } \\
2007 \\
\left(\text { hab/ } \mathrm{Km}^{2}\right)\end{array}$ & $\begin{array}{c}\text { Taux de } \\
\text { croissance } \\
\text { annuel } \\
1999-2007 \\
(\%)\end{array}$ & $\begin{array}{l}\text { Dú au solde } \\
\text { migratoire } \\
\text { apparent }(\%)\end{array}$ & $\begin{array}{l}\text { Dû au solde } \\
\text { naturel }(\%)\end{array}$ & $\begin{array}{c}\text { Evolution } \\
\text { des surfaces } \\
\text { bâties } 1999- \\
2004\end{array}$ \\
\hline Bongheat & 331 & 29,6 & 4,0 & 3,6 & 0,4 & 9,9 \\
\hline Chas & 378 & 107,3 & 3,3 & 2,5 & 0,8 & 11,2 \\
\hline Reignat & 309 & 75,4 & 3,2 & 2,5 & 0,6 & 23,7 \\
\hline $\begin{array}{l}\text { Egliseneuve-Près- } \\
\text { Billom }\end{array}$ & 829 & 49,8 & 2,4 & 2,1 & 0,3 & 9,1 \\
\hline Trézioux & 410 & 23,5 & 2,3 & 2,2 & 0,2 & 9,9 \\
\hline Saint-Julien & 1.076 & 50 & 2,2 & 2,1 & 0,1 & 10,7 \\
\hline Glaine-Montaigut & 536 & 41,5 & 1,3 & 0,9 & 0,4 & 11,5 \\
\hline Montmorin & 562 & 41,2 & 1 & 0,5 & 0,5 & 7,7 \\
\hline Neuville & 332 & 28,7 & 0,8 & 0,5 & 0,3 & 16,6 \\
\hline $\begin{array}{l}\text { Communauté de } \\
\text { communes }\end{array}$ & 11784 & 55 & 1,6 & 1,9 & $-0,3$ & 8,6 \\
\hline $\begin{array}{l}\text { Département du } \\
\text { Puy-de-Dôme }\end{array}$ & 626632 & 78,6 & 0,5 & 0,3 & 0,1 & n.d. \\
\hline
\end{tabular}

Tableau 1 : Indicateurs démographiques des 9 communes étudiées Demographic indicators of the study area 
Les dates de construction des résidences principales témoignent de l'attractivité du territoire : $17 \%$ des logements ont été construits entre 1975 et 1989 et autant après 1990. Malgré tout, les effets induits de cette dynamique résidentielle semblent peu perçus. Du fait de l'organisation du bâti dans la zone, les surfaces artificialisées sont importantes mais dispersées sur un nombre relativement important de bourgs et de hameaux. À l'instar de nombre de périphéries urbaines, le profil sociologique en est modifié en profondeur. Les agriculteurs représentent moins de $5 \%$ de la population totale d'après le recensement de la population de 2007.

Billom-Saint-Dier occupe également une position d'interface entre deux territoires de projets, le ScoT du Pays du Grand Clermont d'une part, et le PNR du Livradois Forez d'autre part (figure 1). Ceci n'est pas sans poser quelques interrogations quant aux modalités d'articulation des politiques portées par l'une et l'autre institution. D'une manière générale, les visions portées sur ce territoire "géré » en commun sont très différenciées. Pour le Parc Naturel Régional Livradois-Forez, Billom-Saint-Dier présente tous les attributs d'un espace de desserrement urbain; c'est le « secteur [...] où la pression urbaine est la plus forte et où la menace d'étalement de l'habitat et des activités ainsi que la banalisation des espaces sont les plus marquées » (Charte du Parc, 2010). La question paysagère est prégnante dans le discours du Parc, et la préservation du cadre de vie et des caractéristiques du paysage est une orientation stratégique forte. À travers sa charte, il préconise et encourage la généralisation des documents de planification et des zonages réglementaires pour préserver les «reliefs structurants » (les Turlurons, buttes représentatives de cette Limagne « montueuse ») et les « espaces agricoles soumis à l'intensification des pratiques et à la pression urbaine ». À travers le paysage voire l'environnement, c'est de la gestion de l'espace agricole dont il s'agit, identifié comme le lieu d'exercice de la planification urbaine.

Cette entrée par le paysage est partagée par les acteurs du ScoT du Grand Clermont, qui portent cependant un regard différent sur ce territoire. Il est considéré comme "un territoire rural vallonné faisant office de transition entre l'agglomération et les reliefs du Livradois » (rapport de présentation du SCoT, 2010). Le diagnostic reconnaît néanmoins
Figure 1 : Situation géographique de Billom-Saint-Dier

Saint-Dier
Location map of Billom-

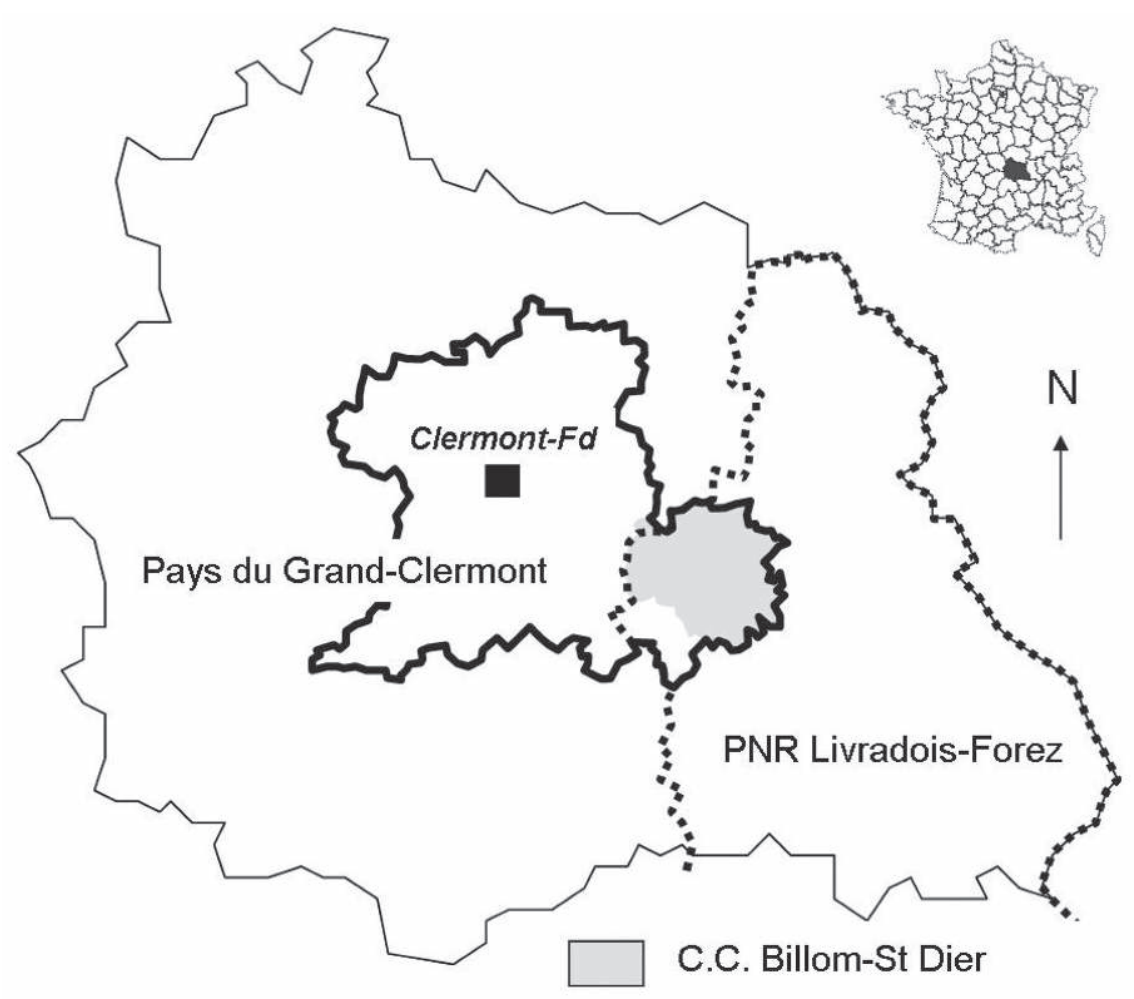


une pression d'urbanisation de plus en plus forte, et le document d'objectifs préconise le maintien d'une " agriculture diversifiée, combinant des espaces en grandes cultures, du pâturage et des forêts " permettant d'entretenir ici la « qualité des paysages ouverts et la conservation d'une biodiversité remarquable» (DOG, 2010).

Cette prise de conscience par les institutions des problématiques associées à la périurbanisation sur ce territoire se traduit par une prise en compte des espaces agricoles dans leurs documents respectifs, où régulation de l'urbanisation et préservation des paysages vont de pair. Cependant, dans les deux cas, l'acteur agricole est peu présent. La protection par zonage, mise en œuvre par les ScoT ou encouragée par l'adoption de documents d'urbanisme par les Parcs, ne prend pas en compte les projets agricoles potentiels ni, a fortiori, les logiques et stratégies (quand celles-ci existent) développées par les acteurs agricoles et plus particulièrement les agriculteurs.

\section{Des signaux faibles de valorisation des composantes productives et commer- ciales des exploitations agricoles de Billom-Saint-Dier}

Dans ce territoire, l'agriculture est une activité encore très présente, tant sur le plan spatial que par le nombre de ses acteurs. Son insertion dans la dynamique urbaine via les projets institutionnels questionne la place des logiques des agriculteurs dans ce contexte. L'adaptation des exploitants agricoles aux dynamiques socio-spatiales et institutionnelles identifiées, peut en être une clé de lecture. Nous avons cherché les signes de cette adaptation des systèmes d'exploitation dans le portefeuille d'activité, dans les systèmes de commercialisation et dans les modalités de gestion du foncier agricole. Un diagnostic agricole a été conduit sur 9 communes de la communauté de communes de BillomSaint-Dier, celles particulièrement concernées par la périurbanisation (figure 2). Il a permis d'identifier 121 exploitations agricoles et leurs caractéristiques productives et commerciales. L'analyse des logiques et des stratégies foncières des agriculteurs a été réalisée à partir d'enquêtes auprès de 36 exploitants.

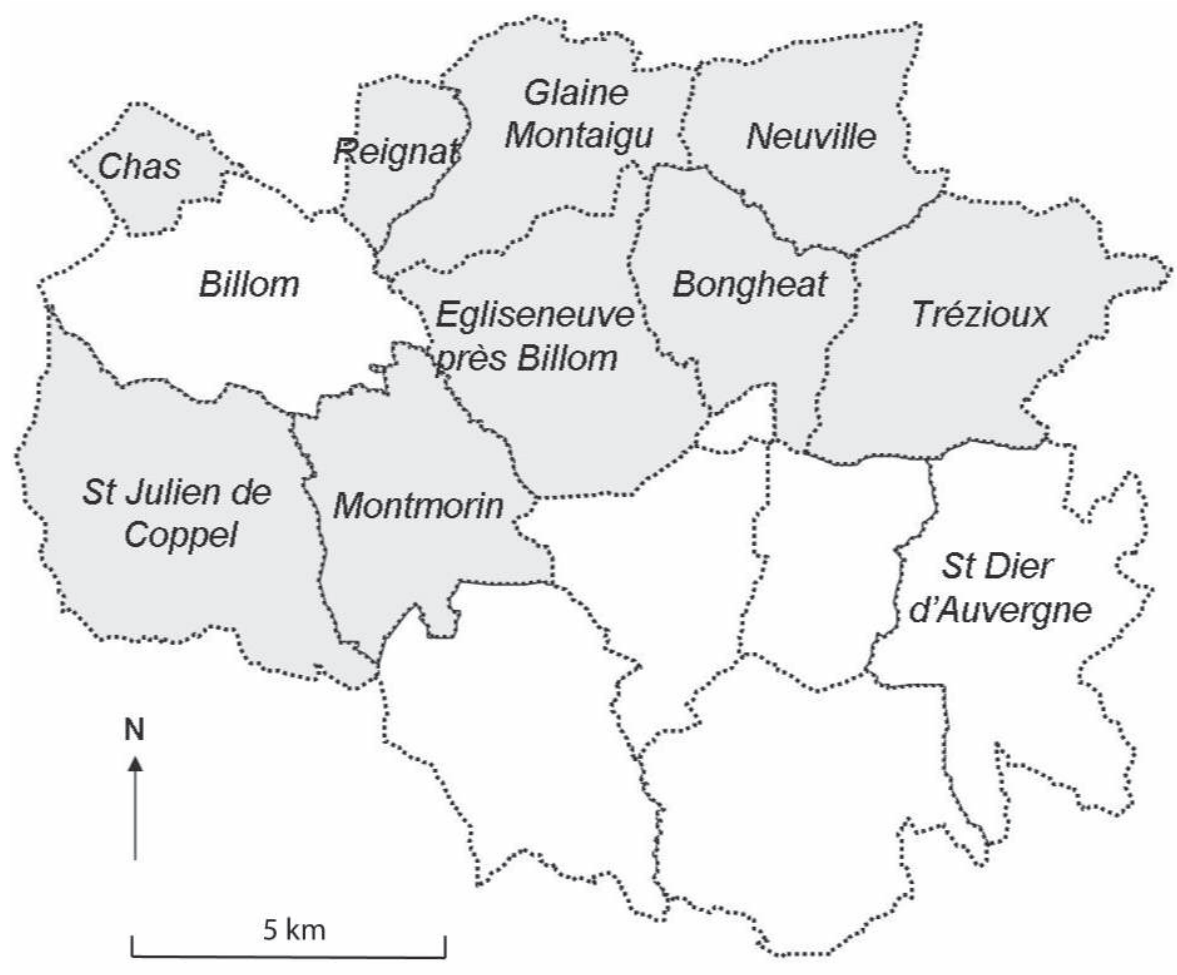

Figure 2: Situation des communes étudiées died municipalities 
L'analyse de l'évolution de l'agriculture sur le territoire de la communauté de communes, de 1970 à nos jours (RGA 1970, 1979, 1988 et 2000), laisse apparaître les grandes tendances visibles à l'échelle nationale (tableau 2) : une diminution générale du nombre d'exploitations agricoles (perte de $65 \%$ des effectifs entre 1970 et 2000); une spécialisation des productions végétales avec en particulier l'augmentation des surfaces en céréales aux dépens de la STH ou des cultures traditionnelles (vigne, ail); une spécialisation des productions animales avec la relative disparition des petits troupeaux ovins et des troupeaux laitiers. Cette évolution se retrouve dans la répartition actuelle des systèmes de production sur les neuf communes étudiées (tableau 3).

\section{La prédominance de systèmes " classiques ", en élevage bovin et/ou grandes cultures, géographiquement déterminés}

Plus des deux tiers des exploitations (68\%) fonctionnent sur les grands systèmes classiques, plus ou moins spécialisés, en élevage ou grandes cultures. La spécialisation en viande bovine concerne le plus fort pourcentage d'exploitations $(28 \%)$ et sa forte présence contraste avec la faible représentation de la spécialisation en élevage bovin lait (3\% des exploitations) et celle des élevages mixtes viande/lait (4\%). Les systèmes spécialisés en grandes cultures concernent, quant à eux, 15 \% des exploitations tan-

\begin{tabular}{|c|c|c|c|c|}
\hline Communauté de communes & 1970 & 1979 & 1988 & 2000 \\
\hline $\mathrm{Nb}$ d'exploitations agricoles & 983 & 746 & 554 & 338 \\
\hline Surface agricole utile (ha) & 13113 & 14129 & 13774 & 13076 \\
\hline dt Terres Labourables & 4457 & 4793 & 4775 & 5074 \\
\hline dt S.T.H. & 8317 & 9120 & 8884 & 7951 \\
\hline dt Vignes & 234 & 154 & 76 & 34 \\
\hline Nombre Vaches laitières & 4295 & 3991 & 2542 & 1278 \\
\hline Nombre Vaches nourrices & 493 & 801 & 1866 & 3333 \\
\hline Nombre Brebis et agnelles & 3799 & 5084 & 4414 & 4308 \\
\hline \multicolumn{5}{|l|}{ Zone d'étude ( 9 communes) } \\
\hline $\mathrm{Nb}$ d'exploitations agricoles & 548 & 418 & 313 & 195 \\
\hline Surface agricole utile (ha) & 7218 & 8043 & 8009 & 7659 \\
\hline dt Terres Labourables & 2720 & 3043 & 3115 & 3498 \\
\hline dt S.T.H. & 4281 & 4861 & 4814 & 4124 \\
\hline dt Vignes & 171 & 110 & 56 & 26 \\
\hline Nombre Vaches laitières & 2376 & 2103 & 1251 & 552 \\
\hline Nombre Vaches nourrices & 176 & 419 & 906 & 1734 \\
\hline Nombre Brebis et agnelles & 2520 & 2928 & 3016 & 3577 \\
\hline
\end{tabular}

Tableau 2 : Indicateurs de l'évolution de l'agriculture à Billom-Saint-Dier (Source Recensements agricoles) Indicators of agriculture evolution in Billom-Saint-Dier 


\begin{tabular}{|l|c|c|c|c|c|}
\hline & $\begin{array}{c}\text { Nombre } \\
\text { d'exploitations }\end{array}$ & $\begin{array}{c}\text { en \% des } \\
\text { exploitations }\end{array}$ & $\begin{array}{c}\text { dont } \\
\text { Double } \\
\text { actifs }\end{array}$ & $\begin{array}{c}\text { SAU } \\
\text { moyenne } \\
\text { toutes } \\
\text { exploitations }\end{array}$ & $\begin{array}{c}\text { SAU moyenne } \\
\text { exploitations } \\
\text { professionnelles }\end{array}$ \\
\hline Grandes cultures & 18 & $15 \%$ & 7 & 68 ha & 107 ha \\
\hline Bovins Viande & 34 & $28 \%$ & 8 & 71 ha & 84 ha \\
\hline Bovins lait & 3 & $3 \%$ & & 46 ha & 46 ha \\
\hline Ovins & 5 & $4 \%$ & 4 & 8 ha & 35 ha \\
\hline & 5 & $4 \%$ & 0 & 83 ha & 83 ha \\
\hline Systèmes Poly-Elevages & 22 & $18 \%$ & 1 & 105 ha & 109 ha \\
\hline $\begin{array}{l}\text { Systèmes Polyculture- } \\
\text { Elevage }\end{array}$ & 15 & $12 \%$ & 4 & 60 ha & 81 ha \\
\hline & 19 & $16 \%$ & 6 & 25 ha & 35 ha \\
\hline Systèmes Mixtes & 121 & $100 \%$ & 30 & 66 ha & 83 ha \\
\hline
\end{tabular}

Tableau 3 : Caractérisation des exploitations agricoles selon les types de systèmes pour les 9 Communes étudiées (sources enquêtes en mairie - 2009)

The different farming systems of the study area

dis que $18 \%$ combinent élevage bovin (laitier ou allaitant) et cultures céréalières dans un système de polyculture élevage. La répartition spatiale de ces systèmes est assez marquée par un gradient altitudinal, et traduit un passage progressif d'une spécialisation à l'autre. Les exploitations spécialisées en grandes cultures qui prédominent au nord, zone la plus basse au contact de la Limagne, laissent progressivement la place à la polyculture élevage, puis plus au Sud, sur les premiers contreforts du Livradois aux systèmes spécialisés en élevage.

Au total, compte tenu de leurs dimensions foncières, ces systèmes représentent près des trois quarts de la surface agricole totale.

Pour chacune de ces trois orientations de production, les surfaces des exploitations sont cependant extrêmement variables : de 4 à 220 ha pour celles en production bovine, de 1 à 175 ha pour celles en cultures. La répartition est assez homogène entre les classes de moins de 20 ha (17\%), de 20 à 50 ha $(27 \%)$, de 50 à 100 ha $(30 \%)$ et supérieures à 100 ha (26\%). La part de la propriété par rapport au fermage diminue à mesure que les surfaces augmentent; générallement, les exploitations dont $100 \%$ de la surface est en propriété sont les plus petites.

Cette diversité des tailles, et en particulier l'existence de « micro exploitations » dans ces systèmes de production classiques surprend. En premier lieu, elle reflète la présence non négligeable d'exploitations en double activité. En second lieu, et au-delà des grandes catégories de spécialisations technico-économiques, elle reflète une diversité de systèmes, dans la combinaison des moyens de production ou dans les modes de valorisation des productions. Derrière une spécialisation « viande bovine ", on trouve ainsi des systèmes variés, du système allaitant caractéristique du Massif central (production de broutards pour la filière italienne) à la production de veau de lait ou l'engraissement de génisses achetées, systèmes diversement exigeants en main-d'œuvre, et diversement rémunérateurs. Dans un même ordre d'idée, nombre d'exploitations en grandes cultures s'appuient sur des productions sous contrat mieux valorisées que dans les filières 
classiques (betteraves, production de semences pour le groupe Limagrain, céréales de qualité panifiable pour l'alimentation humaine, etc.).

\section{La double activité, premier signe d'une agriculture périurbaine}

La proportion d'exploitations en double activité est de $25 \%$. Mais plus que la proportion elle-même, on soulignera les formes de cette pluriactivité. Il s'agit ici d'une double-activité qui présente un caractère périurbain dans la mesure où elle repose principalement sur un emploi salarié, exercé en ville par le chef d'exploitation, en complément de son activité agricole. En fait elle obéit assez souvent à une logique de conservation du patrimoine familial avec poursuite de l'activité agricole des parents aujourd'hui retraités, cela moyennant une simplification du système de production et le repli sur les surfaces en propriété. Dans l'ensemble, ces exploitations sont de plus petite dimension (24 ha en moyenne contre 94 pour les exploitations dites «professionnelles ») et le faire-valoir direct y domine largement. Mais on note également la présence de quelques exploitations de relativement grande dimension (60 à 90 ha). Dans un certain nombre de cas, les parents, encore présents, assurent une part du travail, tandis que le chef d'exploitation lui-même ne réside pas toujours sur place.

Sur le plan de leur fonctionnement technique, ces exploitations ont tendance à prolonger le système pratiqué par les parents. Dès lors, cette double activité se retrouve majoritairement dans les systèmes de production classiques évoqués précédemment. Elle est plus fréquente sur les systèmes céréaliers qu'en élevage, ce qui pourrait s'expliquer par la conjonction d'une organisation plus souple du travail, et d'un moindre éloignement du pôle clermontois.

\section{Les systèmes de production combinatoires : un pas vers la valorisation du périurbain?}

Certaines exploitations (12\% du total) présentent une combinaison plus originale de leur système agricole. Cette combinaison peut recouvrir des productions diverses et concerner à la fois des élevages bovins ou des grandes cultures, associés à un élevage hors-sol ou des petites productions végétales, vigne ou légumes de plein champ (ail, pommes de terre, asperges). Ces exploitations sont présentes dans toutes les communes, exceptées celles situées le plus à l'est, dont les conditions pédologiques et topographiques sont moins propices à ces cultures annexes. Elles ont en moyenne des surfaces plus réduites que les précédentes (67\% ont une surface inférieure à 50 ha). On trouve deux GAEC parmi les très grandes exploitations (4 ont une surface supérieure à 100 ha). Toutes les autres ont un statut d'exploitation individuelle.

Ces combinaisons de productions semblent plus ou moins recouvrir des adaptations des systèmes dominants, au contexte périurbain. Dans certains cas, elles révèlent une stratégie de développement de l'exploitation sur une production ne nécessitant pas de foncier, mais qui reste inscrite dans une filière agro-industrielle (poulaillers industriels en complément de l'élevage bovin ou des cultures de vente). Si elle peut traduire une adaptation à un contexte de relative pression foncière, cette dernière n'est cependant pas toujours liée au contexte périurbain. Sur le territoire étudié, cette stratégie se retrouve en effet sur les communes les plus éloignées du pôle urbain, et les moins sous pression d'urbanisation.

Dans d'autres cas par contre, le facteur de cette localisation périurbaine est plus apparent, sans toutefois peser fortement sur le système. On retrouve alors des combinaisons de productions atypiques comme celle de canards, de plantes médicinales, de bulbes ou encore d'asperges, qui viennent en complément de grandes cultures ou de l'élevage bovin, et dont une partie est commercialisée en vente directe, à la ferme ou sur des marchés locaux. Enfin, cette catégorie révèle également des modes de valorisation basés sur la certification (label Agriculture Biologique, AOP «Côte d'Auvergne » ou projet d'AOP «Ail de Billom »).

\section{Des systèmes spécialisés « atypiques " : une adaptation plus poussée?}

Les spécialisations atypiques concernent $16 \%$ des exploitations. Certaines de ces productions, atypiques pour la zone d'étude, le sont moins à l'échelle nationale (production ovine ou volailles hors-sol). Les autres sont spécialisées dans d'autres petites productions et selon des profils très différents. Deux de ces exploitations sont en vigne. Les agriculteurs 
sont pluriactifs, ont plus de 50 ans, et exploitent de très petites surfaces dont la moitié en fermage. Deux autres, des GAEC, sont en maraîchage (dont une en agriculture biologique), et la dernière est en élevage caprin avec transformation fromagère.

C'est particulièrement dans ces systèmes spécialisés atypiques que l'on retrouve les signes d'une adaptation au contexte périurbain : des innovations, tant en termes de commercialisation (vente à la ferme pour les éleveurs, vente de paniers pour un maraîcher) que de pratique culturale ou d'élevage (système tout herbe ou pratiques biologiques par exemple). Le cas de la vigne illustre la complexité des phénomènes. Production traditionnelle du territoire, elle a longtemps périclité et n'occupe aujourd'hui qu'une portion congrue de l'espace agricole. Elle connaît cependant un regain d'intérêt, et certains exploitants s'appuient sur leur localisation périurbaine pour valoriser leur production. Mais cette relance de la viticulture auvergnate est également portée par un acteur économique puissant, le groupe Limagrain par l'intermédiaire de la coopérative St-Verny, dans une logique de filière plus industrielle avec contractualisation de l'apport des vendanges, et qui ne repose pas sur sa localisation en espace périurbain.

\section{LA GESTION DU FONCIER AGRICOLE PAR LES AGRICULTEURS : DES LOGIQUES PEU MARQUÉES PAR LE CONTEXTE PÉRIURBAIN}

Les logiques de gestion du foncier ont été abordées à l'occasion d'enquêtes auprès d'un échantillon de 36 exploitations. Les agriculteurs sont nombreux à évoquer des difficultés en lien avec des éléments de contexte global, externes au territoire ou à leur exploitation : politique et conjoncture internationale, aléas climatiques, encadrement administratif de leur métier. L'organisation du travail au sein de l'exploitation constitue, dans une moindre mesure, l'une des contraintes pour son développement. Quant aux aspects liés au territoire local, le contexte périurbain apparaît en filigrane : conflits de voisinages avec les « urbains » et surtout, conflits d'usage de l'espace sur l'utilisation des terres (terres reprises aux agriculteurs pour les chevaux ou le bâti). Outre cette difficulté à trouver des terres, les agriculteurs enquêtés abordent parfois le problème de la hausse du prix du foncier mais qu'ils imputent principalement à un phénomène de concurrence entre exploitants. Les dynamiques d'agrandissement (18/36) restent néanmoins dominantes dans l'échantillon enquêté, devant celles de stabilisation du foncier (16/36). L'érosion des surfaces concerne quelques rares exploitations $(2 / 36)$ et relève de situations familiales spécifiques.

\section{Des logiques d'agrandissement pour satisfaire à l'outil de travail}

Tous les exploitants dont le foncier a augmenté depuis l'installation mettent au premier plan la fonction productive de leur activité : il s'agit de " produire pour vivre » ou de « produire pour gagner sa vie », la fonction d'entretien du paysage venant parfois s'ajouter à celle de production. Dans tous les cas, l'augmentation foncière est assimilée véritablement à la satisfaction des besoins de l'outil de travail et les questions de patrimonialisation sont rarement évoquées. L'analyse de l'évolution foncière et des pratiques associées confirme ce point de vue général basé sur la perception que les exploitants présentent de leur métier.

Un premier type d'évolution correspond à des accroissements de surface tout au long de la vie de l'exploitation, par tous les moyens (fermage ou achat). Dans ces cas, les besoins fonciers sont importants pour répondre à des projets de systèmes de production spécialisés ou combinant productions végétales et animales (grandes cultures et bovins). Les exploitants semblent avoir les moyens (financiers, main-d'œuvre) de saisir toutes les opportunités foncières qui s'offrent à eux, à la fois en termes de fermage et d'achat. À l'installation, la surface de ces exploitations variait entre 16 et 105 ha. Depuis, elle a pu doubler voire être multipliée par six et atteint des fourchettes de 84 à 308 ha en 2010 . Toutes ces exploitations bénéficiaient à l'installation d'une bonne assise foncière et d'une durée de vie supérieure à 16 ans. Une grande partie de ces agriculteurs exploite en faire-valoir direct près de la moitié de sa SAU, et pour également une grande partie en fermage familial. D’une manière générale, leur assise foncière apparaît moins précaire.

À l'inverse, la satisfaction des besoins fonciers est plus difficile chez certains agriculteurs. L'augmentation du foncier agricole est ralentie par 
des difficultés financières ou des capacités d'investissement globalement moindres que celles identifiées plus haut. C'est ainsi que l'on voit apparaître des évolutions foncières caractérisées par une augmentation surfacique importante en début de cycle de vie de l'exploitation, suivie d'une phase plus ou moins longue de stabilité. Ces agriculteurs reprennent du foncier "sous condition ». Leur propension au déplacement et à l'éclatement des structures, caractéristique des exploitations agricoles périurbaines (Poulot, 2008), est ici moindre. Mais, il semble que ce soient des logiques davantage contraintes par les capacités d'investissement : le fermage est privilégié à l'achat. Une assise financière couplée à un temps de stabilisation de la structure agricole oriente des évolutions foncières qui interviennent plus tardivement dans le cycle de vie des exploitations.

\section{Une stabilité foncière exprimant des logiques differenciées}

Le dernier type d'évolution foncière se distingue des précédents par la relative stabilité ou un accroissement très faible des surfaces. Plusieurs logiques sous-tendent ce type de dynamique.

Une première relève d'exploitants double actifs : maintenir un équilibre entre activité agricole et activité principale rémunératrice se traduit par une demande faible voire nulle en termes de foncier. Il s'agit d'exploitants installés tardivement, suite à une succession alors qu'ils sont déjà engagés dans une autre activité professionnelle. Le démarrage de l'activité agricole relève alors de motivations de type " passion » ou " préservation du patrimoine », mais reste secondaire. Pour l'ensemble des agriculteurs concernés, la production de denrées alimentaires reste une fonction essentielle de leur rapport au métier d'agriculteur. Ils relient cette fonction à celle d'entretien du paysage ou de préservation du foncier. C'est une façon de « vivre de son revenu, en évitant la course à la prime ».

Une stabilité de la surface foncière peut être aussi un choix contraint; ce sont des logiques d'« évitement du risque » (risque d'endettement par exemple). Il s'agit d'agriculteurs mono-actifs dont l'activité professionnelle tient compte des contraintes familiales ou d'un désir de «ne pas chercher à faire trop ». Ces agriculteurs mettent au pre- mier plan leur fonction de production de denrées alimentaires. Celle-ci peut être attachée à une certaine conception du métier : un rôle important dans la production d'une «bonne alimentation » (la leur et celle des autres) d'une part, et une contribution à la préservation des paysages et de maintien des terres agricoles, d'autre part. Mais ces logiques peuvent également relever d'une rationalité économique, où les agriculteurs visent à maintenir un équilibre entre productivité et revenu. Bien entendu, un manque d'opportunités foncières contraint les évolutions des surfaces agricoles. Il s'agit d'agriculteurs installés dans une situation financière "restreinte ", qui ont manqué d'opportunité, et n'ont pas trouvé de fermage notamment. Apparaissent derrière ces difficultés la concurrence entre exploitants sur le foncier et le difficile accès à la terre pour des exploitants un peu éloignés des réseaux de connaissances des agriculteurs de la zone (personnes installées hors cadre familial et/ou non originaires de la région).

\section{Discussion}

En termes de systèmes de production et de commercialisation, la diversité observée sur notre territoire d'étude rappelle celle identifiée dans d'autres systèmes périurbains (Jarrige, 2004). Cependant, elle reflète en partie la diversité du territoire en question. Mais la diversité de sa géographie physique ne peut à elle seule convaincre des effets de la périurbanisation sur l'adaptation des systèmes de production d'autant que la tendance générale depuis plusieurs années va plutôt à la spécialisation (arrêt de la production laitière, trop contraignante, au profit de l'élevage bovins viande ou de productions hors-sol).

Malgré tout, apparaissent certains signes des effets de la proximité de la ville en termes de valorisation, avec certains cas de vente directe (à la ferme, systèmes des paniers ou insertion dans les AMAP) qui indiquent que certains exploitants modifient leurs pratiques de commercialisation. Quant aux logiques foncières identifiées, elles ne semblent pas correspondre à ce qui est identifié par d'autres auteurs comme un passage généralisé à un intérêt plus patrimonial que productif dans le cas des territoires périurbains (Jarrige et al., 2003). La majorité des logiques observées sur notre territoire d'étude correspond encore à la recherche de satisfaction 
de l'outil de travail et les logiques foncières sont contraintes par les concurrences entre agriculteurs.

On peut s'interroger sur les raisons de la faiblesse des signaux d'adaptation de cette agriculture à son contexte périurbain. Plusieurs éléments de réponse peuvent être suggérés. Notre territoire d'étude constitue la dernière couronne périurbaine de l'agglomération clermontoise, et sa dynamique est de ce fait encore marquée par des déterminants ruraux. Territoire à l'articulation du pays du Grand Clermont et du Parc Naturel Régional LivradoisForez, il fait l'objet de représentations et d'intentions politiques encore très contrastées. Par ailleurs, le processus de périurbanisation s'y est d'abord manifesté par la reprise du bâti ancien de caractère et a, dans un premier temps, peu pesé sur la dynamique foncière. De la même manière, la configuration spatiale du territoire et la dispersion des bourgs et des hameaux influent sur les représentations de l'intensité de l'influence urbaine. L'emprise spatiale de l'urbanisation est distribuée sur plusieurs unités de peuplement. Hormis des extensions pavillonnaires et la construction de lotissements dans les communes les plus proches de Clermont-Ferrand (Chas, Égliseneuve-près-Billom ou Billom), l'urbanisation se fait par petites touches sur un nombre important de hameaux. Ces divers éléments peuvent expliquer dans une certaine mesure le peu d'appropriation de la question agricole par les élus. Celle-ci ne l'est finalement qu'au travers du paysage, ou par l'attention portée aux productions traditionnelles porteuses de culture locale et d'identité territoriale (vigne et ail). Ce manque d'appropriation s'applique également aux agriculteurs s'agissant de la question urbaine.

Finalement, le phénomène périurbain serait-il ici trop récent, trop peu intense ou encore effacé au regard de la conjoncture et de l'ampleur des autres problèmes auxquels les agriculteurs ont à faire face? Cela soulève de nombreuses questions génériques sur les effets de seuil (temporel et/ou d'intensité) au-delà desquels peuvent émerger les signaux d'une adaptation des agriculteurs au contexte périurbain. À partir de quels seuils la valorisation remplace-telle la simple adaptation? À partir de quels seuils des adaptations, innovations, formes de valorisation du contexte apparaissent-elles au niveau des systèmes de production et de commercialisation, au niveau des portefeuilles d'activité, au niveau des modes de gestion du foncier, ou encore des modes de coordinations et de communication entre agriculteurs et avec les autres acteurs du territoire?

Au-delà de ces interrogations, notre travail s'inscrit en complémentarité avec d'autres approches développées sur d'autres agglomérations (Jarrige, 2004 ; Thareau et Le Guen, $2007^{4}$; Soulard et Thareau 2009). Il s'appuie sur les trois clés de lecture de l'agriculture périurbaine que ces travaux suggèrent. Les enquêtes réalisées montrent que c'est surtout la combinaison et l'articulation de ces trois variables portefeuille d'activité, système de production et de commercialisation et gestion du foncier - à l'échelle de l'exploitation, et les déterminants des choix de l'exploitant les concernant, qui révèlent une stratégie d'adaptation au contexte périurbain.

Enfin, si l'adaptation des agriculteurs n'est pas révélée par leurs pratiques commerciales ou de systèmes de production ou encore de gestion du foncier, il convient peut-être de fouiller plus dans les relations sociales entre agriculteurs, dans les dynamiques collectives qui se mettent en place. Certains auteurs émettent l'hypothèse que dans le contexte périurbain, les agriculteurs diversifient leurs interactions sociales, "non seulement dans une visée utilitariste, pour diminuer les conflits potentiels avec leurs voisins, mais plus profondément parce que les transformations des espaces dans lesquels ils exercent leur métier s'accompagnent d'une transformation de leur espace vécu et in fine de la conception de leur métier " (Bernard et al. 2006; Bertrand et al., 2010; Dufour et al., 2003; Souchard, 2010). L'analyse des réseaux de dialogue entre agriculteurs devrait constituer un complément riche à cette première analyse, en permettant d'analyser les configurations sociales dans lesquelles se créent et émergent les nouvelles idées propres aux nouvelles conceptions que les agriculteurs se font de leur métier dans ce contexte particulier : réseaux personnels, clientèle, associations, commissions agricoles communales, etc.

4. Thareau B., Le Guen R., 2007, Être agriculteur près des villes, inventer des modèles professionnels?, communication au colloque « Les agricultures périurbaines, un enjeu pour la ville. Vers des projets de territoires ", Nanterre, 10-12 oct. 2007. 


\section{Bibliographie}

AGRIMONDE, 2009. Agricultures et alimentations du monde en 2050 : scénarios et défis pour un développement durable. Note de synthèse, INRA - CIRAD, 34 p.

Bernard C., Duvernoy I., Dufour A., Albaladejo C., 2006. Les relations sociales des agriculteurs périurbains : quelles articulations au territoire?, Cahiers Agricultures, vol. 15, $\mathrm{n}^{\circ}$ 6, p. 529-534.

Bertrand N., Roussier N., Borg D., 2010. Le rapport de l'agriculture à la ville : vers quelles proximités économiques, dans Bertrand N. (dir.), L'agriculture dans la ville éclatée, Université de Montréal, 2010, p. 47-64.

Bryant C. R., Johnston T. R. R., 1992. Agriculture in the countryside, University of Toronto Press, 232 p.

Bryant C. R., 1997. L'agriculture périurbaine : l'économie politique d'un espace innovateur, Cahiers Agricultures, $n^{\circ}$ 6, p. 125-130.

Cailly L., 2010. La question périurbaine revisitée, dans Cailly L. et Vanier M., La France : une géographie urbaine, Armand Colin, p. 213-230.

Darre J.-P., 1986. Le rôle des réseaux de dialogue entre agriculteurs. Comment les façons de faire et de penser se transforment : l'étude des réseaux de dialogue, Agriscope, $\mathrm{n}^{\circ} 7$, p. 143-151.

Debarbieux B., 2005. Obsolescence ou actualité des objets géographiques modernes? À propos de la ville, de la campagne, du périurbain et de quelques autres objets conventionnels, dans Arlaud S., Jean Y., Royoux D. (ed.), Rural-urbain, Nouveaux lieux, Nouvelles frontières, PUR, p. 33-43.

Derruau M., 1949. La grande Limagne auvergnate et bourbonnaise. Étude géographique, Delaunay, 540 p.

Dufour A., Bernard C., Angelucci M.-A., 2003. Reconstruction des identités professionnelles autour de la multifonctionnalité de l'agriculture, l'exemple des coteaux du Lyonnais, Ruralia, n 12/13 [http://ruralia.revues.org/document334.html].

Duvernoy I., 2002. Espace agricole périurbain et politiques communales d'aménagement : l'exemple de l'agglomération albigeoise, Cybergeo, 14 p.

Duvernoy I., Jarrige F., Moustier P., Serrano J., 2005. Une agriculture multifonctionnelle dans le projet urbain : quelle reconnaissance? Quelle gouvernance?, Les Cahiers de la Multifonctionnalité, $\mathrm{n}^{\circ}$ 8, p. 87-104.

FEL A., 1983. Le massif Central, Flammarion, 346 p.

Germain P., Le Guen R., Thareau B., 2006. La re-territorialisation du développement agricole : le cas de l'agriculture périurbaine d'Angers, Revue d'Économie Régionale et Urbaine, $\mathrm{n}^{\circ}$ 3, p. 373-392.

Gumuchian H., Pecqueur B., 2007. La ressource territoriale, Paris, Economica, $252 \mathrm{p}$.

Jarrige F., 2004. Les mutations d'une agriculture méditerranéenne face à la croissance urbaine : dynamique et enjeux autour de Montpellier, Cahiers Agricultures, vol. 13, $\mathrm{n}^{\circ} 1$, p. 64-74.

Jarrige F., Jouve A.-M., Napoleone C., 2003. Et si le capitalisme patrimonial foncier changeait nos paysages quo- tidiens?, Courrier de l'environnement de l'INRA, $\mathrm{n}^{\circ} 49$, p. 13-28.

Jarrige F., Thinon P., Delay C., Montfraix P., 2009. L'agriculture s'invite dans le projet urbain. Le schéma de cohérence territoriale de Montpellier Agglomération, Innovations Agronomiques, $\mathrm{n}^{\circ}$ 5, p. 41-51.

Loudiy S., Lardon S., Lelli L., 2010. Can Agriculture Be a Territorial Resource in Periurban territories? The Case of an inter-municipal structure 'Volvic Sources et Volcans, dans Galli M., Lardon S., Marraccini E., Bonari (ed.), Agricultural management in peri-urban areas. The experience of an international workshop, edizioni ETS, p. 67-81.

Loudiy S., Maury C., Lardon S., 2011 . Agriculture(s) et projet urbain durable : issues incertaines, destinées imprévisibles, VertigO, vol. 11, n 2, mis en ligne le 22 janvier 2012 [http:// vertigo.revues.org/11481].

Pasini I., Minelli A., Fleury A., 2012. Initiatives agricoles comme antiprojet d'un projet d'urbanistes, Projets de Paysage $\mathrm{n}^{\circ} 7,9 \mathrm{p}$. [http://www.projetsdepaysage.fr/fr/initiatives_ agricoles_comme_antiprojet_d_un_projet_d_urbanistes].

Poulot M., 2008. Le retour de l'agriculture dans la ville élargie : vers la durabilité des territoires, Habilitation à diriger des recherches, Université Paris Ouest Nanterre, 253 p.

Poulot M., 2011. 2011. Des arrangements autour de l'agriculture en périurbain : du lotissement agricole au projet de territoire, vol. $11, \mathrm{n}^{\circ} 2$, mis en ligne le 4 octobre 2011 [http://vertigo.revues.org/11188].

Rouget N., 2008. Les dynamiques agricoles dans les espaces urbains et périurbains. Diversification et stratégies d'adaptation des agricultures. Les cas des périphéries Sud-Est de Lille et Nord de Lens, Thèse de géographie, Université Paris Ouest - Nanterre La Défense, 456 p.

Souchard N., 2010. Incertitudes et productions identitaires agricoles : vers un éclatement des logiques d'action?, dans Bertrand N. (dir), L'agriculture dans la ville éclatée, Université de Montréal, p. 99-121.

Soulard C., Thareau B., 2009. Les exploitations agricoles périurbaines : diversité et logiques de développement, Innovations Agronomiques $\mathrm{n}^{\circ}$ 5, p 27-40.

Vianey G., 2005. Entre conception de l'exercice du métier et représentation de l'activité agricole en périurbain : esquisse d'une analyse des logiques foncières, Les Cahiers de la Multifonctionnalité, $\mathrm{n}^{\circ}$ 8, p. 105-114.

Vianey G., Bacconnier-Baylet S., Duvernoy I., 2006. L'aménagement communal périurbain : maintenir l'agriculture pour préserver quelle ruralité?, Revue d'économie régionale et urbaine, $\mathrm{n}^{\circ} 3$, p. 355-372.

Vidal R., Fleury A., 2009. La place de l'agriculture dans la métropole verte. Nostalgies, utopies et réalités dans l'aménagement des territoires aux franges urbaines, Projets de Paysage, $15 \mathrm{p}$.

[http://www.projetsdepaysage.fr/fr/la_place_de_l_agriculture_ dans_la_metropole_verte]. 